\title{
Quantitative uniqueness for second order elliptic operators with strongly singular coefficients
}

\section{Ching-Lung Lin, Gen Nakamura and Jenn-Nan Wang}

\begin{abstract}
In this paper we study the local behavior of a solution to second order elliptic operators with sharp singular coefficients in lower order terms. One of the main results is the bound on the vanishing order of the solution, which is a quantitative estimate of the strong unique continuation property. Our proof relies on Carleman estimates with carefully chosen phases. A key strategy in the proof is to derive doubling inequalities via three-sphere inequalities. Our method can also be applied to certain elliptic systems with similar singular coefficients.
\end{abstract}

\section{Introduction}

Assume that $\Omega$ is a connected open set containing 0 in $\mathbb{R}^{n}$ for $n \geq 2$. Let

$$
P(x, D)=\sum_{j, k} a_{j k}(x) D_{j} D_{k}
$$

be an elliptic differential operator in $\Omega$ such that $a_{j k}(0)$ is a real symmetric matrix and $a_{j k}(x)$ is Lipschitz continuous in $\Omega$, where $D_{j}=\partial / \partial x_{j}$, $j=1, \ldots, n$. Note that $a_{j k}(x)$ could be complex valued at $x \neq 0$. In this paper we consider the following second order differential inequality:

$$
|P(x, D) u| \leq \frac{C_{1}}{|x|^{2}}|u|+\frac{C_{2}}{|x|}|\nabla u| \quad \text { in } \quad \Omega,
$$

where $C_{2}$ is sufficiently small. Before proceeding to the main discussion, we want to point out that restrictions described above are necessary. It is 
well known that the Lipschitz smoothness requirement on $a_{i j}$ is minimal for the unique continuation to hold [14]. Counterexamples given by Alinhac [2] show that the restriction of $a_{i j}(0)$ being real is necessary for the strong unique continuation. On the other hand, regarding the constant $C_{2}$, the strong unique continuation fails for (1.1) if $C_{2}$ is not small, see [3] and [16]. Finally, simple counterexamples also show that the singular coefficients on the right side of (1.1) are sharp for the strong unique continuation. Under the same assumptions, the strong unique continuation property for (1.1) was proved by Regbaoui [15]. But Regbaoui did not give any quantitative estimate on the vanishing order of $u$ satisfying (1.1). This is our main goal in this work. The development of qualitative unique continuation property has a long history. We do not intend to give a summary here. We refer to the paper [10] and references therein for more details.

Concerning about the quantitative estimate of the uniqueness for partial differential operators, we would like to mention several related works. Using the frequency function, Garofala and Lin [5, 6] derived a quantitative version of the strong unique continuation for strongly second order elliptic operators. In [5], they also considered $|x|^{-2}$ potentials but without first order terms. In [6], they studied full lower order terms with certain singular coefficients, but they are not sharp. Also in [11], Kukavica used the frequency function to prove the maximal vanishing order of solutions to the strong second order elliptic operator with essentially bounded potentials. Our method in this paper is different from those in [5], [6], and [11]. Our key tools are Carleman estimates. Besides of the difference in method, the differential operator $P(x, D)$ in $(1.1)$ is only elliptic and the coefficients on the right hand side of (1.1) are strongly singular. None of [5], [6], and [11] dealt with the equation as (1.1).

On the other hand, Donnelly and Fefferman [4] applied Carleman's technique to derive the maximal vanishing order of the eigenfunction with respect to the corresponding eigenvalue on a compact smooth Riemannian manifold. Also, in [12], Lin applied the Carleman estimate proved by Jerison and Kenig [9] to derive a quantitative estimate of the strong unique continuation property for the Schrödinger equation with $L_{l o c}^{n / 2}$ potential. However, the methods in [4] and [12] can not be applied to (1.1) with strongly singular coefficients. The difficulty lies in the fact that all Carleman estimates used to treat the strong unique continuation contain only polynomial weights, which are not "singular" enough to handle sharp singular coefficients in the lower derivatives. In this work, we overcome this difficulty by deriving threesphere inequalities using slightly singular than polynomial weights. Then we proceed to derive doubling inequalities and the bound on the vanishing order of the solution to (1.1) by applying three-sphere inequalities recursively. 
In this paper, for brevity, we only consider the scalar second order elliptic operator. But our method can also be applied to the case where $P(x, D)$ is an elliptic system as

$$
P(x, D)=\operatorname{diag}\left(P_{1}(x, D), \ldots, P_{\ell}(x, D)\right),
$$

where $P_{j}(x, D), j=1, \ldots, \ell$, are second order elliptic operators with Lipschitz coefficients and satisfy that $P_{j}(0, D)=\cdots=P_{\ell}(0, D)$ with real symmetric coefficients. All methods mentioned above do not seem to work in this general case. Finally, we would like to mention that quantitative estimates of the strong unique continuation are useful in studying the nodal sets of eigenfunctions [4], or solutions of second order elliptic equations [7], [13], or the inverse problem [1]. The main results of the paper are summarized as follows. Assume that $B_{R_{0}} \subset \Omega$.

Theorem 1.1 There exists a positive number $R_{1}<1$ such that if $0<r_{1}<$ $r_{2}<r_{3} \leq R_{0}$ and $r_{1} / r_{3}<r_{2} / r_{3}<R_{1}$, then

$$
\int_{|x|<r_{2}}|u|^{2} d x \leq C\left(\int_{|x|<r_{1}}|u|^{2} d x\right)^{\tau}\left(\int_{|x|<r_{3}}|u|^{2} d x\right)^{1-\tau}
$$

for $u \in H^{1}\left(B_{R_{0}}\right)$ satisfying (1.1) in $B_{R_{0}}$, where $C$ and $0<\tau<1$ depend on $r_{1} / r_{3}, r_{2} / r_{3}$ and $P(x, D)$.

Remark 1.1 From the proof, it suffices to take $R_{1} \leq 1 / 4$. Moreover, the constants $C$ and $\tau$ can be explicitly written as $C=\max \left\{C_{0}\left(r_{2} / r_{1}\right)^{n}, \exp \left(B \beta_{0}\right)\right\}$ and $\tau=B /(A+B)$, where $C_{0}>1$ and $\beta_{0}$ are constants depending on $P(x, D)$ and

$$
\begin{aligned}
& A=A\left(r_{1} / r_{3}, r_{2} / r_{3}\right)=\left(\log \left(r_{1} / r_{3}\right)-1\right)^{2}-\left(\log \left(r_{2} / r_{3}\right)\right)^{2}, \\
& B=B\left(r_{2} / r_{3}\right)=-1-2 \log \left(r_{2} / r_{3}\right) .
\end{aligned}
$$

The explicit forms of these constants are important in the proof of Theorem 1.2.

Theorem 1.2 There exists a constant $C$ depending on $P(x, D)$ such that if $u \in H_{\text {loc }}^{1}(\Omega)$ is a nonzero solution to (1.1) with $C_{2}<C$, then we can find a constant $R_{2}$ depending on $P(x, D)$ and a constant $m_{1}$ depending on $P(x, D)$ and $\|u\|_{L^{2}\left(|x|<R_{2}^{2}\right)} /\|u\|_{L^{2}\left(|x|<R_{2}^{4}\right)}$ satisfying

$$
\int_{|x|<R}|u|^{2} d x \geq K R^{m_{1}}
$$

where $R$ is sufficiently small and the constant $K$ depends on $n, R_{2}$ and $u$. 
In view of the standard unique continuation property for (1.1) in a connected domain containing the origin, if $u$ vanishes in a neighborhood of the origin then it vanishes identically in $\Omega$. Theorem 1.2 provides an upper bound on the vanishing order of a nontrivial solution to (1.1). The following doubling inequality is another quantitative estimate of the strong unique continuation for (1.1).

Theorem 1.3 Let $u \in H_{l o c}^{1}(\Omega)$ be a nonzero solution to (1.1). Then there exist positive constants $R_{3}$ and $C_{3}$ depending on $P(x, D), m_{1}$ such that if $0<r \leq R_{3}$, then

$$
\int_{|x| \leq 2 r}|u|^{2} d x \leq C_{3} \int_{|x| \leq r}|u|^{2} d x,
$$

where $m_{1}$ is the constant obtained in Theorem 1.2.

The rest of the paper is devoted to the proofs of Theorem 1.1-1.3.

\section{Proof of Theorem 1.1}

This section is devoted to the proof of Theorem 1.1. To begin, we recall a Carleman estimate with weight $\varphi_{\beta}=\varphi_{\beta}(|x|)=\exp \left(\frac{\beta}{2}(\log |x|)^{2}\right)$ derived in $[15]$.

Lemma 2.1 [15, Theorem 1.2] For any $\beta>0$ large enough. Let $S$ be a small neighborhood of 0 and $u: S \backslash\{0\} \subset \Omega \rightarrow \mathbb{R}, u \in C_{0}^{\infty}(S \backslash\{0\})$. Then we have

$$
\begin{aligned}
\beta^{3} \int \varphi_{\beta}^{2}|x|^{-n}|u|^{2} d x+ & \beta \int \varphi_{\beta}^{2}|x|^{-n+2}|\nabla u|^{2} d x \leq \\
& \leq \tilde{C}_{0} \int \varphi_{\beta}^{2}|x|^{-n+4}|P(x, D) u|^{2} d x
\end{aligned}
$$

for some positive constant $\tilde{C}_{0}$ depending only on $P(x, D)$.

Remark 2.1 The estimate (2.1) in Lemma 2.1 remains valid if we assume $u \in H^{2}(S \backslash\{0\})$ with compact support. This can be easily obtained by cutting off $u$ for small $|x|$ and regularizing.

We now proceed to the main part of the proof. Using regularization, Friedrich's lemma, and ellipticity of $P(x, D)$, we can see that if $u \in H_{l o c}^{1}(\Omega)$ satisfies (1.1) then $u \in H_{l o c}^{2}(\Omega \backslash\{0\})$. To begin, we first consider the case 
where $0<r_{1}<r_{2}<R<1$ and $B_{R} \subset \Omega$. The constant $R$ will be determined later. To use the Carleman estimate (2.1), we need to cut-off $u$. So let $\xi(x) \in C_{0}^{\infty}\left(\mathbb{R}^{n}\right)$ satisfy $0 \leq \xi(x) \leq 1$ and

$$
\xi(x)= \begin{cases}0, & |x| \leq r_{1} / e \\ 1, & r_{1} / 2<|x|<e r_{2}, \\ 0, & |x| \geq 3 r_{2}\end{cases}
$$

Here $e=\exp (1)$. It is easy to see that for all multiindex $\alpha$

$$
\left\{\begin{array}{l}
\left|D^{\alpha} \xi\right|=O\left(r_{1}^{-|\alpha|}\right) \text { for all } r_{1} / e \leq|x| \leq r_{1} / 2 \\
\left|D^{\alpha} \xi\right|=O\left(r_{2}^{-|\alpha|}\right) \text { for all } e r_{2} \leq|x| \leq 3 r_{2}
\end{array}\right.
$$

On the other hand, repeating the proof of Corollary 17.1.4 in [8], we can show that

$$
\left.\left.\int_{a_{1} r<|x|<a_{2} r}|| x\right|^{|\alpha|} D^{\alpha} u\right|^{2} d x \leq C^{\prime} \int_{a_{3} r<|x|<a_{4} r}|u|^{2} d x, \quad|\alpha| \leq 2,
$$

for all $0<a_{3}<a_{1}<a_{2}<a_{4}$ such that $B_{a_{4} r} \subset \Omega$, where the constant $C^{\prime}$ is independent of $r$.

Noting that the commutator $[P(x, D), \xi]$ is a first order differential operator. Applying (2.1) to $\xi u$ and using (1.1), (2.2), (2.3) implies

$$
\begin{aligned}
\beta^{3} \int_{r_{1} / 2<|x|<e r_{2}} \varphi_{\beta}^{2}|x|^{-n}|u|^{2} d x+\beta \int_{r_{1} / 2<|x|<e r_{2}} \varphi_{\beta}^{2}|x|^{-n+2}|\nabla u|^{2} d x \leq \\
\leq \beta^{3} \int \varphi_{\beta}^{2}|x|^{-n}|\xi u|^{2} d x+\beta \int \varphi_{\beta}^{2}|x|^{-n+2}|\nabla(\xi u)|^{2} d x \\
\leq \tilde{C}_{0} \int \varphi_{\beta}^{2}|x|^{-n+4}|P(x, D)(\xi u)|^{2} d x \\
\leq \tilde{C}_{0} \int \varphi_{\beta}^{2}\left(C_{1}^{2}|x|^{-n}|\xi u|^{2}+C_{2}^{2}|x|^{-n+2}|\xi \nabla u|^{2}\right) d x \\
+\tilde{C}_{0} \int_{\beta}^{2}|x|^{-n+4}|[P(x, D), \xi] u|^{2} d x \\
\leq \tilde{C}_{1}\left\{\int_{r_{1} / 2<|x|<e r_{2}} \varphi_{\beta}^{2}|x|^{-n}|u|^{2} d x+\int_{r_{1} / 2<|x|<e r_{2}} \varphi_{\beta}^{2}|x|^{-n+2}|\nabla u|^{2} d x\right. \\
\quad+\int_{r_{1} / e<|x|<r_{1} / 2} \varphi_{\beta}^{2}|x|^{-n}|u|^{2} d x+\int_{r_{1} / e<|x|<r_{1} / 2} \varphi_{\beta}^{2}|x|^{-n+2}|\nabla u|^{2} d x \\
\left.\quad+\int_{e r_{2}<|x|<3 r_{2}} \varphi_{\beta}^{2}|x|^{-n}|u|^{2} d x+\int_{e r_{2}<|x|<3 r_{2}} \varphi_{\beta}^{2}|x|^{-n+2}|\nabla u|^{2} d x\right\}
\end{aligned}
$$




$$
\begin{aligned}
\leq & \tilde{C}_{2}\left\{\int_{r_{1} / 2<|x|<e r_{2}} \varphi_{\beta}^{2}|x|^{-n}|u|^{2} d x+\int_{r_{1} / 2<|x|<e r_{2}} \varphi_{\beta}^{2}|x|^{-n+2}|\nabla u|^{2} d x\right. \\
& +r_{1}^{-n} \varphi_{\beta}^{2}\left(r_{1} / e\right) \int_{r_{1} / e<|x|<r_{1} / 2}\left(|u|^{2}+\left.\left.|| x\right|^{2} \nabla u\right|^{2}\right) d x \\
& \left.+r_{2}^{-n} \varphi_{\beta}^{2}\left(e r_{2}\right) \int_{e r_{2}<|x|<3 r_{2}}\left(|u|^{2}+\left.\left.|| x\right|^{2} \nabla u\right|^{2}\right) d x\right\} \\
\leq & \tilde{C}_{3}\left\{\int_{r_{1} / 2<|x|<e r_{2}} \varphi_{\beta}^{2}|x|^{-n}|u|^{2} d x+\int_{r_{1} / 2<|x|<e r_{2}} \varphi_{\beta}^{2}|x|^{-n+2}|\nabla u|^{2} d x\right. \\
& \left.+r_{1}^{-n} \varphi_{\beta}^{2}\left(r_{1} / e\right) \int_{r_{1} / 4<|x|<r_{1}}|u|^{2} d x+r_{2}^{-n} \varphi_{\beta}^{2}\left(e r_{2}\right) \int_{2 r_{2}<|x|<4 r_{2}}|u|^{2} d x\right\}
\end{aligned}
$$

where $\tilde{C}_{1}, \tilde{C}_{2}$, and $\tilde{C}_{3}$ are independent of $r_{1}$ and $r_{2}$. Now letting $\beta_{0} \geq 1$ and $\beta \geq \beta_{0} \geq 2 \tilde{C}_{3}$ in (2.4), we immediately get that

$$
\begin{aligned}
& \int_{r_{1} / 2<|x|<e r_{2}} \varphi_{\beta}^{2}|x|^{-n}|u|^{2} d x+\int_{r_{1} / 2<|x|<e r_{2}} \varphi_{\beta}^{2}|x|^{-n+2}|\nabla u|^{2} d x \leq \\
& \quad \leq \tilde{C}_{4}\left\{r_{1}^{-n} \varphi_{\beta}^{2}\left(r_{1} / e\right) \int_{r_{1} / 4<|x|<r_{1}}|u|^{2} d x+r_{2}^{-n} \varphi_{\beta}^{2}\left(e r_{2}\right) \int_{2 r_{2}<|x|<4 r_{2}}|u|^{2} d x\right\}
\end{aligned}
$$

where $\tilde{C}_{4}=1 / \tilde{C}_{3}$. It follows easily from $(2.5)$ that

$$
\begin{aligned}
& r_{2}^{-n} \varphi_{\beta}^{2}\left(r_{2}\right) \int_{r_{1} / 2<|x|<r_{2}}|u|^{2} d x \leq \\
& \quad \leq \int_{r_{1} / 2<|x|<e r_{2}} \varphi_{\beta}^{2}|x|^{-n}|u|^{2} d x \\
& \quad \leq \tilde{C}_{4}\left\{r_{1}^{-n} \varphi_{\beta}^{2}\left(r_{1} / e\right) \int_{r_{1} / 4<|x|<r_{1}}|u|^{2} d x+r_{2}^{-n} \varphi_{\beta}^{2}\left(e r_{2}\right) \int_{2 r_{2}<|x|<4 r_{2}}|u|^{2} d x\right\} .
\end{aligned}
$$

Dividing $r_{2}^{-n} \varphi_{\beta}^{2}\left(r_{2}\right)$ on the both sides of (2.6) implies

$$
\begin{aligned}
\int_{r_{1} / 2<|x|<r_{2}}|u|^{2} d x \leq & \tilde{C}_{4}\left\{\left(r_{2} / r_{1}\right)^{n}\left[\varphi_{\beta}^{2}\left(r_{1} / e\right) / \varphi_{\beta}^{2}\left(r_{2}\right)\right] \int_{r_{1} / 4<|x|<r_{1}}|u|^{2} d x\right. \\
& \left.+\left[\varphi_{\beta}^{2}\left(e r_{2}\right) / \varphi_{\beta}^{2}\left(r_{2}\right)\right] \int_{2 r_{2}<|x|<4 r_{2}}|u|^{2} d x\right\} \\
\leq & \tilde{C}_{5}\left\{\left(r_{2} / r_{1}\right)^{n}\left[\varphi_{\beta}^{2}\left(r_{1} / e\right) / \varphi_{\beta}^{2}\left(r_{2}\right)\right] \int_{|x|<r_{1}}|u|^{2} d x\right. \\
& \left.+\left(r_{2} / r_{1}\right)^{n}\left[\varphi_{\beta}^{2}\left(e r_{2}\right) / \varphi_{\beta}^{2}\left(r_{2}\right)\right] \int_{|x|<4 r_{2}}|u|^{2} d x\right\},
\end{aligned}
$$

where $\tilde{C}_{5}=\max \left\{\tilde{C}_{4}, 1\right\}$. 
With such choice of $\tilde{C}_{5}$, we see that

$$
\tilde{C}_{5}\left(r_{2} / r_{1}\right)^{n}\left[\varphi_{\beta}^{2}\left(r_{1} / e\right) / \varphi_{\beta}^{2}\left(r_{2}\right)\right]>1
$$

for all $0<r_{1}<r_{2}$. Adding $\int_{|x|<r_{1} / 2}|u|^{2} d x$ to both sides of (2.7) and choosing $r_{2} \leq 1 / 4$, we obtain that

$$
\begin{aligned}
\int_{|x|<r_{2}}|u|^{2} d x \leq & 2 \tilde{C}_{5}\left(r_{2} / r_{1}\right)^{n}\left[\varphi_{\beta}^{2}\left(r_{1} / e\right) / \varphi_{\beta}^{2}\left(r_{2}\right)\right] \int_{|x|<r_{1}}|u|^{2} d x \\
& +2 \tilde{C}_{5}\left(r_{2} / r_{1}\right)^{n}\left[\varphi_{\beta}^{2}\left(e r_{2}\right) / \varphi_{\beta}^{2}\left(r_{2}\right)\right] \int_{|x|<1}|u|^{2} d x .
\end{aligned}
$$

For simplicity, by denoting

$$
\begin{aligned}
& A=\beta^{-1} \log \left[\varphi_{\beta}^{2}\left(r_{1} / e\right) / \varphi_{\beta}^{2}\left(r_{2}\right)\right]=\left(\log r_{1}-1\right)^{2}-\left(\log r_{2}\right)^{2}>0, \\
& B=-\beta^{-1} \log \left[\varphi_{\beta}^{2}\left(e r_{2}\right) / \varphi_{\beta}^{2}\left(r_{2}\right)\right]=-1-2 \log r_{2}>0,
\end{aligned}
$$

(2.8) becomes

$$
\begin{aligned}
& \int_{|x|<r_{2}}|u|^{2} d x \leq \\
& \quad \leq 2 \tilde{C}_{5}\left(r_{2} / r_{1}\right)^{n}\left\{\exp (A \beta) \int_{|x|<r_{1}}|u|^{2} d x+\exp (-B \beta) \int_{|x|<1}|u|^{2} d x\right\} .
\end{aligned}
$$

To further simplify the terms on the right hand side of (2.9), we consider two cases. If

$$
\exp \left(A \beta_{0}\right) \int_{|x|<r_{1}}|u|^{2} d x<\exp \left(-B \beta_{0}\right) \int_{|x|<1}|u|^{2} d x
$$

then we can pick a $\beta>\beta_{0}$ such that

$$
\exp (A \beta) \int_{|x|<r_{1}}|u|^{2} d x=\exp (-B \beta) \int_{|x|<1}|u|^{2} d x .
$$

Using such $\beta$, we obtain from (2.9) that

$$
\begin{aligned}
\int_{|x|<r_{2}}|u|^{2} d x & \leq 4 \tilde{C}_{5}\left(r_{2} / r_{1}\right)^{n} \exp (A \beta) \int_{|x|<r_{1}}|u|^{2} d x= \\
& =4 \tilde{C}_{5}\left(r_{2} / r_{1}\right)^{n}\left(\int_{|x|<r_{1}}|u|^{2} d x\right)^{\frac{B}{A+B}}\left(\int_{|x|<1}|u|^{2} d x\right)^{\frac{A}{A+B}} .
\end{aligned}
$$

On the other hand, if

$$
\exp \left(-B \beta_{0}\right) \int_{|x|<1}|u|^{2} d x \leq \exp \left(A \beta_{0}\right) \int_{|x|<r_{1}}|u|^{2} d x
$$


then we have

$$
\begin{aligned}
\int_{|x|<r_{2}}|u|^{2} d x & \leq\left(\int_{|x|<1}|u|^{2} d x\right)^{\frac{B}{A+B}}\left(\int_{|x|<1}|u|^{2} d x\right)^{\frac{A}{A+B}} \\
& \leq \exp \left(B \beta_{0}\right)\left(\int_{|x|<r_{1}}|u|^{2} d x\right)^{\frac{B}{A+B}}\left(\int_{|x|<1}|u|^{2} d x\right)^{\frac{A}{A+B}} .
\end{aligned}
$$

Putting together $(2.10),(2.11)$, and setting $\tilde{C}_{6}=\max \left\{4 \tilde{C}_{5}\left(r_{2} / r_{1}\right)^{n}, \exp \left(B \beta_{0}\right)\right\}$, we arrive at

$$
\int_{|x|<r_{2}}|u|^{2} d x \leq \tilde{C}_{6}\left(\int_{|x|<r_{1}}|u|^{2} d x\right)^{\frac{B}{A+B}}\left(\int_{|x|<1}|u|^{2} d x\right)^{\frac{A}{A+B}} .
$$

Now for the general case, we take $R_{1} \leq 1 / 4$ and consider $0<r_{1}<r_{2}<r_{3}$ with $r_{1} / r_{3}<r_{2} / r_{3} \leq 1 / 4$. By scaling, i.e. defining $\widehat{u}(y):=u\left(r_{3} y\right)$ and $\widehat{a_{i j}}(y)=a_{i j}\left(r_{3} y\right)$, we derive from (2.12) that

$$
\int_{|y|<r_{2} / r_{3}}|\widehat{u}|^{2} d y \leq C\left(\int_{|y|<r_{1} / r_{3}}|\widehat{u}|^{2} d y\right)^{\tau}\left(\int_{|y|<1}|\widehat{u}|^{2} d y\right)^{1-\tau},
$$

where $\tau=B /(A+B)$ with

$$
\begin{aligned}
& A=A\left(r_{1} / r_{3}, r_{2} / r_{3}\right)=\left(\log \left(r_{1} / r_{3}\right)-1\right)^{2}-\left(\log \left(r_{2} / r_{3}\right)\right)^{2}, \\
& B=B\left(r_{2} / r_{3}\right)=-1-2 \log \left(r_{2} / r_{3}\right),
\end{aligned}
$$

and $C=\max \left\{4 \tilde{C}_{5}\left(r_{2} / r_{1}\right)^{n}, \exp \left(B \beta_{0}\right)\right\}$. We want to remark that $\tilde{C}_{5}$ can be chosen independent of the scaling factor $r_{3}$ provided $r_{3}<1$. Restoring the variable $x=r_{3} y$ in (2.13) gives

$$
\int_{|x|<r_{2}}|u|^{2} d x \leq C\left(\int_{|x|<r_{1}}|u|^{2} d x\right)^{\tau}\left(\int_{|x|<r_{3}}|u|^{2} d x\right)^{1-\tau} .
$$

The proof now is complete.

\section{Proof of Theorem 1.2 and Theorem 1.3}

In this section, we prove Theorem 1.2 and Theorem 1.3. Without loss of generality, we assume $P(0, D)=\Delta$ by the change of coordinates. We begin with another Carleman estimate derived in [15, Lemma 2.1]: for any $u \in$ $C_{0}^{\infty}\left(\mathbb{R}^{n} \backslash\{0\}\right)$ and for any $m \in\left\{j+\frac{1}{2}, j \in \mathbb{N}\right\}$ we have

$$
\sum_{|\alpha| \leq 2} \int m^{2-2|\alpha|}|x|^{-2 m+2|\alpha|-n}\left|D^{\alpha} u\right|^{2} d x \leq C \int|x|^{-2 m+4-n}|\Delta u|^{2} d x
$$

where $C$ only depends on the dimension $n$. 
Remark 3.1 Using the cut-off function and regularization, estimate (3.1) remains valid for any fixed $m$ if $u \in H_{l o c}^{2}\left(\mathbb{R}^{n} \backslash\{0\}\right)$ with compact support.

In view of Remark 3.1, we can apply (3.1) to the function $\chi u$ with $\chi(x) \in$ $C_{0}^{\infty}\left(\mathbb{R}^{n} \backslash\{0\}\right)$. Therefore, we define $\chi(x) \in C_{0}^{\infty}\left(\mathbb{R}^{n} \backslash\{0\}\right)$ such that

$$
\chi(x)= \begin{cases}0 & \text { if } \quad|x| \leq \delta / 3 \\ 1 & \text { in } \quad \delta / 2 \leq|x| \leq\left(R_{0}+1\right) R_{0} R / 4=r_{4} R \\ 0 & \text { if } \quad 2 r_{4} R \leq|x|\end{cases}
$$

where $\delta \leq R_{0}^{2} R / 4, R_{0}>0$ is a small number which will be chosen later and $R$ is sufficiently small satisfying $0<R \leq R_{0}$. Here the number $R$ is not yet fixed and is given by $R=(\gamma m)^{-1}$, where $\gamma>0$ is a large constant which will be chosen later. Using the estimate (3.1) and the equation (1.1), we can derive that

$$
\begin{aligned}
& \sum_{|\alpha| \leq 2} m^{2-2|\alpha|} \int_{\delta / 2 \leq|x| \leq r_{4} R}|x|^{-2 m+2|\alpha|-n}\left|D^{\alpha} u\right|^{2} d x \\
& \leq \sum_{|\alpha| \leq 2} m^{2-2|\alpha|} \int|x|^{-2 m+2|\alpha|-n}\left|D^{\alpha}(\chi u)\right|^{2} d x \leq C \int|x|^{-2 m+4-n}|\Delta(\chi u)|^{2} d x \\
& \leq C \int_{\delta / 2 \leq|x| \leq r_{4} R}|x|^{-2 m+4-n}|\Delta u|^{2} d x+C \int_{|x|>r_{4} R}|x|^{-2 m+4-n}|\Delta(\chi u)|^{2} d x \\
& +C \int_{\delta / 3 \leq|x| \leq \delta / 2}|x|^{-2 m+4-n}|\Delta(\chi u)|^{2} d x \\
& \leq \hat{C}^{\prime} \int_{\delta / 2 \leq|x| \leq r_{4} R}|x|^{-2 m+4-n}|\Delta u-P(x, D) u|^{2} d x \\
& +\hat{C}^{\prime} \int_{\delta / 2 \leq|x| \leq r_{4} R}|x|^{-2 m+4-n}|P(x, D) u|^{2} d x \\
& +C \int_{|x|>r_{4} R}|x|^{-2 m+4-n}|\Delta(\chi u)|^{2} d x+C \int_{\delta / 3 \leq|x| \leq \delta / 2}|x|^{-2 m+4-n}|\Delta(\chi u)|^{2} d x \\
& \leq C^{\prime} \sum_{|\alpha|=2} r_{4}^{2} R^{2} \int_{\delta / 2 \leq|x| \leq r_{4} R}|x|^{-2 m+4-n}\left|D^{\alpha} u\right|^{2} d x \\
& +C^{\prime} C_{1}^{2} \int_{\delta / 2 \leq|x| \leq r_{4} R}|x|^{-2 m-n}|u|^{2} d x+C^{\prime} C_{2}^{2} \sum_{|\alpha|=1} \int_{\delta / 2 \leq|x| \leq r_{4} R}|x|^{-2 m+2-n}\left|D^{\alpha} u\right|^{2} d x \\
& +C \int_{|x|>r_{4} R}|x|^{-2 m+4-n}|\Delta(\chi u)|^{2} d x+C \int_{\delta / 3 \leq|x| \leq \delta / 2}|x|^{-2 m+4-n}|\Delta(\chi u)|^{2} d x,
\end{aligned}
$$

where the constant $C^{\prime}$ depends on $n$. 
By carefully checking terms on both sides of (3.2), we now choose $\gamma=$ $\sqrt{C^{\prime}}$ and thus

$$
R=\frac{1}{\gamma m}=\frac{1}{\sqrt{C^{\prime}} m} \quad \text { and } \quad r_{4}^{2} R^{2}=\frac{R_{0}^{2}\left(R_{0}+1\right)^{2}}{16 m^{2} C^{\prime}} .
$$

Hence, choosing $R_{0}<1$ (suffices to guarantee $R_{0}^{2}\left(R_{0}+1\right)^{2} / 16<1 / 2$ ), $m \geq \tilde{m}_{0}=\tilde{m}_{0}\left(R_{0}\right)$, and $C_{2}$ sufficiently small such that

$$
\frac{1}{\sqrt{C^{\prime}} m} \leq R_{0}, \quad \frac{m^{2}}{2}>C^{\prime} C_{1}^{2}, \quad \text { and } \quad 1-C^{\prime} C_{2}^{2}>\frac{1}{2},
$$

we can remove the first three terms on the right hand side of the last inequality in (3.2) and obtain

$$
\begin{aligned}
& \sum_{|\alpha| \leq 2} m^{2-2|\alpha|} \int_{\delta / 2<|x|<r_{4} R}|x|^{-2 m+2|\alpha|-n}\left|D^{\alpha} u\right|^{2} d x \leq \\
& \leq 2 C \int_{\delta / 3<|x|<\delta / 2}|x|^{-2 m+4-n}|\Delta(\chi u)|^{2} d x \\
&+2 C \int_{r_{4} R<|x|<2 r_{4} R}|x|^{-2 m+4-n}|\Delta(\chi u)|^{2} d x
\end{aligned}
$$

In view of the definition of $\chi$, it is easy to see that for all multiindex $\alpha$

$$
\left\{\begin{array}{l}
\left|D^{\alpha} \chi\right|=O\left(\delta^{-|\alpha|}\right) \text { for all } \delta / 3<|x|<\delta / 2, \\
\left|D^{\alpha} \chi\right|=O\left(\left(r_{4} R\right)^{-|\alpha|}\right) \text { for all } r_{4} R<|x|<2 r_{4} R .
\end{array}\right.
$$

Note that $R_{0}^{2} \leq r_{4}$ provided $R_{0} \leq 1 / 15$. Therefore, using (3.4) and (2.3) in (3.3), we derive

$$
\begin{aligned}
m^{2}(2 \delta)^{-2 m-n} & \int_{\delta / 2<|x| \leq 2 \delta}|u|^{2} d x+m^{2}\left(R_{0}^{2} R\right)^{-2 m-n} \int_{2 \delta<|x| \leq R_{0}^{2} R}|u|^{2} d x \leq \\
\leq & \sum_{|\alpha| \leq 2} m^{2-2|\alpha|} \int_{\delta / 2<|x|<r_{4} R}|x|^{-2 m+2|\alpha|-n}\left|D^{\alpha} u\right|^{2} d x \\
\leq & \tilde{C} \sum_{|\alpha| \leq 2} \delta^{-4+2|\alpha|} \int_{\delta / 3<|x|<\delta / 2}|x|^{-2 m+4-n}\left|D^{\alpha} u\right|^{2} d x \\
& +C^{\prime \prime} \sum_{|\alpha| \leq 2}\left(r_{4} R\right)^{-4+2|\alpha|} \int_{r_{4} R<|x|<2 r_{4} R}|x|^{-2 m+4-n}\left|D^{\alpha} u\right|^{2} d x \\
\leq & \tilde{C}^{\prime}(\delta / 3)^{-2 m-n} \int_{|x| \leq \delta}|u|^{2} d x+C^{\prime \prime}\left(r_{4} R\right)^{-2 m-n} \int_{|x| \leq R_{0} R}|u|^{2} d x
\end{aligned}
$$

where $\tilde{C}^{\prime}$ and $C^{\prime \prime}$ are independent of $R_{0}, R$, and $m$. 
We then add $m^{2}(2 \delta)^{-2 m-n} \int_{|x| \leq \delta / 2}|u|^{2} d x$ to both sides of (3.5) and obtain

$$
\begin{aligned}
& \frac{1}{2} m^{2}(2 \delta)^{-2 m-n} \int_{|x| \leq 2 \delta}|u|^{2} d x+m^{2}\left(R_{0}^{2} R\right)^{-2 m-n} \int_{|x| \leq R_{0}^{2} R}|u|^{2} d x= \\
&= \frac{1}{2} m^{2}(2 \delta)^{-2 m-n} \int_{|x| \leq 2 \delta}|u|^{2} d x+m^{2}\left(R_{0}^{2} R\right)^{-2 m-n} \int_{|x| \leq 2 \delta}|u|^{2} d x \\
&+m^{2}\left(R_{0}^{2} R\right)^{-2 m-n} \int_{2 \delta<|x| \leq R_{0}^{2} R}|u|^{2} d x \\
& \leq \frac{1}{2} m^{2}(2 \delta)^{-2 m-n} \int_{|x| \leq 2 \delta}|u|^{2} d x+\frac{1}{2} m^{2}(2 \delta)^{-2 m-n} \int_{|x| \leq 2 \delta}|u|^{2} d x \\
&+m^{2}\left(R_{0}^{2} R\right)^{-2 m-n} \int_{2 \delta<|x| \leq R_{0}^{2} R}|u|^{2} d x \\
& \leq\left(\tilde{C}^{\prime}+m^{2}\right)(\delta / 3)^{-2 m-n} \int_{|x| \leq \delta}|u|^{2} d x+C^{\prime \prime}\left(r_{4} R\right)^{-2 m-n} \int_{|x| \leq R_{0} R}|u|^{2} d x \\
&=\left(\tilde{C}^{\prime}+m^{2}\right)(\delta / 3)^{-2 m-n} \int_{|x| \leq \delta}|u|^{2} d x \\
& \quad+m^{2}\left(R_{0}^{2} R\right)^{-2 m-n} C^{\prime \prime} m^{-2}\left(\frac{R_{0}^{2}}{r_{4}}\right)^{2 m+n} \int_{|x| \leq R_{0} R}|u|^{2} d x .
\end{aligned}
$$

We first observe that

$$
\begin{aligned}
C^{\prime \prime} m^{-2}\left(\frac{R_{0}^{2}}{r_{4}}\right)^{2 m+n} & =C^{\prime \prime} m^{-2}\left(\frac{4 R_{0}}{R_{0}+1}\right)^{2 m+n} \\
& \leq C^{\prime \prime} m^{-2}\left(4 R_{0}\right)^{2 m+n} \leq \exp (-2 m)
\end{aligned}
$$

for all $R_{0} \leq 1 / 16$ and $m^{2} \geq C^{\prime \prime}$. Thus, we obtain that

$$
\begin{aligned}
\frac{1}{2} m^{2}(2 \delta)^{-2 m-n} \int_{|x| \leq 2 \delta}|u|^{2} d x & +m^{2}\left(R_{0}^{2} R\right)^{-2 m-n} \int_{|x| \leq R_{0}^{2} R}|u|^{2} d x \leq \\
\leq & \left(\tilde{C}^{\prime}+m^{2}\right)(\delta / 3)^{-2 m-n} \int_{|x| \leq \delta}|u|^{2} d x \\
& +m^{2}\left(R_{0}^{2} R\right)^{-2 m-n} \exp (-2 m) \int_{|x| \leq R_{0} R}|u|^{2} d x .
\end{aligned}
$$

It should be noted that (3.7) is valid for all $m=j+\frac{1}{2}$ with $j \in \mathbb{N}$ and $j \geq j_{0}$, where $j_{0}$ depends on $R_{0}$. Setting $R_{j}=\left(\gamma\left(j+\frac{1}{2}\right)\right)^{-1}$ and using the 
relation $m=(\gamma R)^{-1}$, we get from (3.7) that

$$
\begin{aligned}
\frac{1}{2} m^{2}(2 \delta)^{-2 m-n} & \int_{|x| \leq 2 \delta}|u|^{2} d x+m^{2}\left(R_{0}^{2} R_{j}\right)^{-2 m-n} \int_{|x| \leq R_{0}^{2} R_{j}}|u|^{2} d x \leq \\
\leq & \left(\tilde{C}^{\prime}+m^{2}\right)(\delta / 3)^{-2 m-n} \int_{|x| \leq \delta}|u|^{2} d x \\
& +m^{2}\left(R_{0}^{2} R_{j}\right)^{-2 m-n} \exp \left(-2 c R_{j}^{-1}\right) \int_{|x| \leq R_{0} R_{j}}|u|^{2} d x
\end{aligned}
$$

for all $j \geq j_{0}$ and $c=\gamma^{-1}$. We now observe that

$$
R_{j+1}<R_{j}<2 R_{j+1} \text { for all } j \in \mathbb{N} .
$$

Thus, if $R_{j+1}<R \leq R_{j}$, we can conclude that

$$
\left\{\begin{array}{l}
\int_{|x| \leq R_{0}^{2} R}|u|^{2} d x \leq \int_{|x| \leq R_{0}^{2} R_{j}}|u|^{2} d x \\
\exp \left(-2 c R_{j}^{-1}\right) \int_{|x| \leq R_{0} R_{j}}|u|^{2} d x \leq \exp \left(-c R^{-1}\right) \int_{|x| \leq R}|u|^{2} d x
\end{array}\right.
$$

where we have used the inequality $R_{0} R_{j} \leq 2 R_{j+1} / 16<R_{j+1}$ to derive the second inequality above. Namely, we have from (3.8) and (3.9) that

$$
\begin{aligned}
\frac{1}{2} m^{2}(2 \delta)^{-2 m-n} & \int_{|x| \leq 2 \delta}|u|^{2} d x+m^{2}\left(R_{0}^{2} R_{j}\right)^{-2 m-n} \int_{|x| \leq R_{0}^{2} R}|u|^{2} d x \leq \\
\leq & \left(\tilde{C}^{\prime}+m^{2}\right)(\delta / 3)^{-2 m-n} \int_{|x| \leq \delta}|u|^{2} d x \\
& +m^{2}\left(R_{0}^{2} R_{j}\right)^{-2 m-n} \exp \left(-c R^{-1}\right) \int_{|x| \leq R}|u|^{2} d x
\end{aligned}
$$

If there exists $s \in \mathbb{N}$ such that

$$
R_{j+1}<R_{0}^{2 s} \leq R_{j} \quad \text { for some } j \geq j_{0},
$$

then replacing $R$ by $R_{0}^{2 s}$ in (3.10) leads to

$$
\begin{aligned}
& \frac{1}{2} m^{2}(2 \delta)^{-2 m-n} \int_{|x| \leq 2 \delta}|u|^{2} d x+m^{2}\left(R_{0}^{2} R_{j}\right)^{-2 m-n} \int_{|x| \leq R_{0}^{2 s+2}}|u|^{2} d x \leq \\
& \leq\left(\tilde{C}^{\prime}+m^{2}\right)(\delta / 3)^{-2 m-n} \int_{|x| \leq \delta}|u|^{2} d x \\
& +m^{2}\left(R_{0}^{2} R_{j}\right)^{-2 m-n} \exp \left(-c R_{0}^{-2 s}\right) \int_{|x| \leq R_{0}^{2 s}}|u|^{2} d x .
\end{aligned}
$$


Here $s$ and $R_{0}$ are yet to be determined. The trick now is to find suitable $s$ and $R_{0}$ satisfying (3.11) and the inequality

$$
\exp \left(-c R_{0}^{-2 s}\right) \int_{|x| \leq R_{0}^{2 s}}|u|^{2} d x \leq \frac{1}{2} \int_{|x| \leq R_{0}^{2 s+2}}|u|^{2} d x
$$

holds with such choices of $s$ and $R_{0}$.

It is time to use the three-sphere inequality (1.2). To this end, we choose $r_{1}=R_{0}^{2 k+2}, r_{2}=R_{0}^{2 k}$ and $r_{3}=R_{0}^{2 k-2}$ for $k \geq 1$. Note that $r_{1} / r_{3}<r_{2} / r_{3} \leq$ $R_{0}^{2} \leq 1 / 4$. Thus (1.2) implies

$$
\int_{|x|<R_{0}^{2 k}}|u|^{2} d x / \int_{|x|<R_{0}^{2 k+2}}|u|^{2} d x \leq C^{1 / \tau}\left(\int_{|x|<R_{0}^{2 k-2}}|u|^{2} d x / \int_{|x|<R_{0}^{2 k}}|u|^{2} d x\right)^{a}
$$

where

$$
C=\max \left\{C_{0} R_{0}^{-2 n}, \exp \left(\beta_{0}\left(-1-4 \log R_{0}\right)\right)\right\}
$$

and

$$
\begin{aligned}
a=\frac{1-\tau}{\tau}=\frac{A}{B} & =\frac{\left(\log \left(r_{1} / r_{3}\right)-1\right)^{2}-\left(\log \left(r_{2} / r_{3}\right)\right)^{2}}{-1-2 \log \left(r_{2} / r_{3}\right)} \\
& =\frac{\left(4 \log R_{0}-1\right)^{2}-\left(2 \log R_{0}\right)^{2}}{-1-4 \log R_{0}} .
\end{aligned}
$$

It is not hard to see that

$$
\left\{\begin{array}{l}
1<C \leq C_{0} R_{0}^{-\beta_{1}} \\
2<a \leq-4 \log R_{0}
\end{array}\right.
$$

where $\beta_{1}=\max \left\{2 n, 4 \beta_{0}\right\}$. Combining (3.15) and using (3.14) recursively, we have that

$$
\begin{aligned}
\int_{|x| \leq R_{0}^{2 s}}|u|^{2} d x / \int_{|x| \leq R_{0}^{2 s+2}}|u|^{2} d x & \leq C^{1 / \tau}\left(\int_{|x|<R_{0}^{2 s-2}}|u|^{2} d x / \int_{|x|<R_{0}^{2 s}}|u|^{2} d x\right)^{a} \\
& \leq C^{\frac{a^{s-1}-1}{\tau(a-1)}}\left(\int_{|x|<R_{0}^{2}}|u|^{2} d x / \int_{|x|<R_{0}^{4}}|u|^{2} d x\right)^{a^{s-1}}
\end{aligned}
$$

for all $s \geq 1$. Now from the definition of $a$, we have $\tau=1 /(a+1)$ and thus

$$
\frac{a^{s-1}-1}{\tau(a-1)}=\frac{a+1}{a-1}\left(a^{s-1}-1\right) \leq 3 a^{s-1} .
$$


Then it follows from (3.16) that

$$
\begin{aligned}
& \int_{|x| \leq R_{0}^{2 s}}|u|^{2} d x / \int_{|x| \leq R_{0}^{2 s+2}}|u|^{2} d x \leq \\
& \quad \leq C^{3\left(-4 \log R_{0}\right)^{s-1}}\left(\int_{|x|<R_{0}^{2}}|u|^{2} d x / \int_{|x|<R_{0}^{4}}|u|^{2} d x\right)^{a^{s-1}} \\
& \quad \leq\left(C_{0}^{3}\left(R_{0}\right)^{-3 \beta_{1}}\right)^{\left(-4 \log R_{0}\right)^{s-1}}\left(\int_{|x|<R_{0}^{2}}|u|^{2} d x / \int_{|x|<R_{0}^{4}}|u|^{2} d x\right)^{a^{s-1}} .
\end{aligned}
$$

Thus, by (3.17), we can get that

$$
\begin{aligned}
& \exp \left(-c R_{0}^{-2 s}\right) \int_{|x| \leq R_{0}^{2 s}}|u|^{2} d x \leq \exp \left(-c R_{0}^{-2 s}\right)\left(C_{0}^{3}\left(R_{0}\right)^{-3 \beta_{1}}\right)^{\left(-4 \log R_{0}\right)^{s-1}} \\
& \cdot\left(\int_{|x|<R_{0}^{2}}|u|^{2} d x / \int_{|x|<R_{0}^{4}}|u|^{2} d x\right)^{a^{s-1}} \int_{|x| \leq R_{0}^{2 s+2}}|u|^{2} d x .
\end{aligned}
$$

Let $\mu=-\log R_{0}$, then if $R_{0}(\leq 1 / 16)$ is sufficiently small, i.e., $\mu$ is sufficiently large, we can see that

$$
2 t \mu>(t-1) \log (4 \mu)+\log \left(\log C_{0}^{3}+3 \beta_{1} \mu\right)-\log (c / 4)
$$

for all $t \in \mathbb{N}$. In other words, we have that for $R_{0}$ small

$$
\left(C_{0}^{3} R_{0}^{-3 \beta_{1}}\right)^{\left(-4 \log R_{0}\right)^{t-1}}<\exp \left(c R_{0}^{-2 t} / 4\right)<(1 / 2) \exp \left(c R_{0}^{-2 t} / 2\right)
$$

for all $t \in \mathbb{N}$. We now fix such $R_{0}$ so that (3.19) holds. The constants $m_{0}\left(R_{0}\right)$ and $j_{0}\left(R_{0}\right)$ are fixed as well. It is a key step in our proof that we can find a universal constant $R_{0}$. After fixing $R_{0}$, we then define a number $t_{0}$ as

$$
\begin{array}{r}
t_{0}=\inf \left\{t \in \mathbb{R}: t \geq\left(\log 2-\log (a c)+\log \log \left(\int_{|x|<R_{0}^{2}}|u|^{2} d x / \int_{|x|<R_{0}^{4}}|u|^{2} d x\right)\right)\right. \\
\left.\times\left(-2 \log R_{0}-\log a\right)^{-1}\right\} .
\end{array}
$$

It should be noted that $t_{0}$ depends on $R_{0}$ and $N$, where

$$
N=\left(\int_{|x|<R_{0}^{2}}|u|^{2} d x / \int_{|x|<R_{0}^{4}}|u|^{2} d x\right) .
$$

By (3.15), one can easily check that $-2 \log R_{0}-\log a>0$ for all $R_{0} \leq 1 / 16$. With the choice of $t_{0}$, we can see that

$$
\left(\int_{|x|<R_{0}^{2}}|u|^{2} d x / \int_{|x|<R_{0}^{4}}|u|^{2} d x\right)^{a^{t-1}} \leq \exp \left(c R_{0}^{-2 t} / 2\right)
$$

for all $t \geq t_{0}$. 
Let $s_{1}$ be the smallest positive integer such that $s_{1} \geq t_{0}$. If

$$
R_{0}^{2 s_{1}} \leq R_{j_{0}}=\left(\gamma\left(j_{0}+1 / 2\right)\right)^{-1}
$$

then we can find a $j_{1} \in \mathbb{N}$ with $j_{1} \geq j_{0}$ such that (3.11) holds, i.e.,

$$
R_{j_{1}+1}<R_{0}^{2 s_{1}} \leq R_{j_{1}}
$$

On the other hand, if

$$
R_{0}^{2 s_{1}}>R_{j_{0}}
$$

then we pick the smallest positive integer $s_{2}>s_{1}$ such that $R_{0}^{2 s_{2}} \leq R_{j_{0}}$ and thus we can also find a $j_{1} \in \mathbb{N}$ with $j_{1} \geq j_{0}$ for which (3.11) holds. We now define $s$, depending on $P(x, D)$ and $N$, as

$$
s=\left\{\begin{array}{llll}
s_{1} & \text { if } & (3.21) & \text { holds } \\
s_{2} & \text { if } & (3.22) & \text { holds }
\end{array}\right.
$$

It is important to note that with such $s,(3.11)$ is satisfied for some $j_{1}$ (depending on $P(x, D)$ and $N)$ and (3.19), (3.20) hold. Therefore, we set $m_{1}=n+2\left(j_{1}+1 / 2\right)$ and $m=\left(m_{1}-n\right) / 2$, where $m_{1}$ and $m$ depend on $P(x, D)$ and $N$. Combining (3.18), (3.19) and (3.20) yields that

$$
\begin{aligned}
\exp \left(-c R_{0}^{-2 s}\right) & \int_{|x| \leq R_{0}^{2 s}}|u|^{2} d x \leq \\
\leq & \exp \left(-c R_{0}^{-2 s}\right)\left(C_{0}^{3}\left(R_{0}\right)^{-3 \beta_{1}}\right)^{\left(-3 \log R_{0}\right)^{s-1}} \\
& \cdot\left(\int_{|x|<R_{0}^{2}}|u|^{2} d x / \int_{|x|<R_{0}^{4}}|u|^{2} d x\right)^{a^{(s-1)}} \int_{|x| \leq R_{0}^{2 s+2}}|u|^{2} d x \\
\leq & \frac{1}{2} \int_{|x| \leq R_{0}^{2 s+2}}|u|^{2} d x
\end{aligned}
$$

which is (3.13). Using (3.13) in (3.12), we have that

$$
\begin{aligned}
\frac{1}{2} m^{2}(2 \delta)^{-2 m-n} \int_{|x| \leq 2 \delta}|u|^{2} d x & +\frac{1}{2} m^{2}\left(R_{0}^{2} R_{j_{1}}\right)^{-2 m-n} \int_{|x| \leq R_{0}^{2 s+2}}|u|^{2} d x \leq \\
& \leq\left(\tilde{C}^{\prime}+m^{2}\right)(\delta / 3)^{-2 m-n} \int_{|x| \leq \delta}|u|^{2} d x .
\end{aligned}
$$

From (3.23), we get that

$$
\frac{\left(m_{1}-n\right)^{2}}{8 \tilde{C}^{\prime}+2\left(m_{1}-n\right)^{2}}\left(3 R_{0}^{2} R_{j_{1}}\right)^{-m_{1}} \int_{|x| \leq R_{0}^{2 s+2}}|u|^{2} d x \leq \delta^{-m_{1}} \int_{|x| \leq \delta}|u|^{2} d x
$$


and

$$
\frac{1}{2} m^{2}(2 \delta)^{-2 m-n} \int_{|x| \leq 2 \delta}|u|^{2} d x \leq\left(\tilde{C}^{\prime}+m^{2}\right)(\delta / 3)^{-2 m-n} \int_{|x| \leq \delta}|u|^{2} d x
$$

which implies

$$
\int_{|x| \leq 2 \delta}|u|^{2} d x \leq \frac{8 \tilde{C}^{\prime}+2\left(m_{1}-n\right)^{2}}{\left(m_{1}-n\right)^{2}} 6^{m_{1}} \int_{|x| \leq \delta}|u|^{2} d x .
$$

The estimates (3.24) and (3.25) are valid for all $\delta \leq R_{0}^{2 s+2} / 4$. Therefore, (1.3) holds with $R_{2}=R_{0}$. (1.4) holds with $R_{3}=R_{0}^{2 s+2} / 8$ and $C_{3}=$ $\frac{8 \tilde{C}^{\prime}+2\left(m_{1}-n\right)^{2}}{\left(m_{1}-n\right)^{2}} 6^{m_{1}}$ and the proof is now complete.

\section{References}

[1] Alessandrini, G., Beretta, E., Rosset, E. and Vessella, S.: Optimal stability for inverse elliptic boundary value problems with unknow boundaries. Ann. Scuola Norm. Sup. Pisa Cl. Sci. 29 (2000), no. 4, 755-806.

[2] Alinhac, A.: Non-unicité pour des opérateurs différentiels à caractéristiques complexes simples. Ann. Sci. École Norm. Sup. (4) 13 (1980), no. 3, 385-393.

[3] Alinhac, S. And Baouendi, M. S.: A counterexample to strong uniqueness for partial differential equations of Schrödinger's type. Comm. Partial Differential Equations 19 (1994), no. 9-10, 1727-1733.

[4] Donnelly, H. and Fefferman, C.: Nodal sets of eigenfunctions on Riemannian manifolds. Invent. Math. 93 (1988), no. 1, 161-183.

[5] Garofalo, N. and Lin, F. H.: Monotonicity properties of variational integrals, $A_{p}$ weights and unique continuation. Indiana Univ. Math. J. 35 (1986), no. 2, 245-267.

[6] Garofalo, N. and Lin, F. H.: Unique continuation for elliptic operators: a geometric-variational approach. Comm. Pure Appl. Math. 40 (1987), no. $3,347-366$.

[7] Hardt, R. And Simon, L.: Nodal sets for solutions of elliptic equations. J. Differential Geom. 30 (1989), no. 2, 505-522.

[8] Hörmander, L.: The analysis of linear partial differential operators. III. Grundlehren der Mathematischen Wissenschaften 274. Springer-Verlag, Berlin, 1985.

[9] Jerison, D. And Kenig, C.: Unique continuation and absence of positive eigenvalues for Schrodinger operators. Ann. of Math. (2) 121 (1985), no. 3, 463-494. 
[10] Koch, H. and Tataru, D.: Carleman estimates and unique continuation for second-order elliptic equations with nonsmooth coefficients. Comm. Pure Appl. Math. 54 (2001), no. 3, 339-360.

[11] KukavicA, I.: Quantitative uniqueness for second order elliptic operators. Duke Math. J. 91 (1998), no. 2, 225-240.

[12] Lin, F. H.: A uniqueness theorem for parabolic equations. Comm. Pure Appl. Math. 43 (1990), no. 1, 127-136.

[13] Lin, F.H.: Nodal sets of solutions of elliptic and parabolic equations. Comm. Pure Appl. Math. 44 (1991), no. 3, 287-308.

[14] Pliš, A.: On non-uniqueness in Cauchy problem for an elliptic second order differential equation. Bull. Acad. Polon. Sci. Sér. Sci. Math. Astronom. Phys. 11 (1963), 95-100.

[15] Regbaoui, R.: Strong uniqueness for second order differential operators. J. Differential Equations 141 (1997), no. 2, 201-217.

[16] Wolff, T.: A counterexample in a unique continuation problem. Comm. Anal. Geom. 2 (1994), no. 1, 79-102.

Recibido: 18 de diciembre de 2008

Ching-Lung Lin

Department of Mathematics

NCTS (Tainan)

National Cheng Kung University

Tainan 70131, Taiwan

cllin2@mail.ncku.edu.tw

Gen Nakamura

Department of Mathematics

Hokkaido University

Sapporo 060-0810, Japan

gnaka@math.sci.hokudai.ac.jp

Jenn-Nan Wang

Department of Mathematics

Taida Institute of Mathematical Sciences, NCTS (Taipei)

National Taiwan University

Taipei 106, Taiwan

jnwang@math.ntu.edu.tw

The first and third authors are partially supported by the National Science Council of Taiwan. the second author is partially supported by Grant-in-Aid for Scientific Research (B)(2) (No. 19340028) of Japan Society for Promotion of Science. 